Revue des patrimoines

\title{
Camille Enlart s'en va-t-en guerre. Le musée de Sculpture comparée pendant la Première Guerre mondiale
}

Jean-Marc Hofman

\section{OpenEdition}

Journals

Édition électronique

URL : http://journals.openedition.org/insitu/10894

DOI : 10.4000/insitu.10894

ISSN : 1630-7305

Éditeur

Ministère de la Culture

\section{Référence électronique}

Jean-Marc Hofman, «Camille Enlart s'en va-t-en guerre. Le musée de Sculpture comparée pendant la Première Guerre mondiale », In Situ [En ligne], 23 | 2014, mis en ligne le 21 février 2014, consulté le 15 novembre 2019. URL : http://journals.openedition.org/insitu/10894

Ce document a été généré automatiquement le 15 novembre 2019.

\section{(c) (†) $\ominus$}

In Situ Revues des patrimoines est mis à disposition selon les termes de la licence Creative Commons Attribution - Pas d'Utilisation Commerciale - Pas de Modification 4.0 International. 


\section{Camille Enlart s'en va-t-en guerre. Le musée de Sculpture comparée pendant la Première Guerre mondiale}

Jean-Marc Hofman

La guerre a fait notre patriotisme plus conscient et la
fureur jalouse de nos ennemis, en s'acharnant sur les
cuuvres d'art, les a rendues plus chères à ceux-là
mêmes que les gloires artistiques de la France
laissaient indifférents.
Camille Enlart, revue Les Arts, 1919

1 Tout au long du premier conflit mondial, Camille Enlart n'eut de cesse de dénoncer les exactions commises à l'encontre du patrimoine architectural et artistique ${ }^{1}$. Archéologue et médiéviste reconnu, directeur du musée de Sculpture comparée au Trocadéro, à Paris, cet érudit s'y employa avec une détermination sans faille et une opiniâtreté vengeresse dès le mois de septembre 1914, au lendemain des bombardements de la cathédrale Notre-Dame de Reims.

2 Son premier geste fort, Camille Enlart l'accomplit incontestablement en 1914, en tant que président de la Société des Amis des cathédrales. Bénéficiant de l'appui de la Société française d'archéologie, cette association avait été fondée deux ans plus tôt afin de « faciliter l'audition et l'étude des œuvres d'art d'inspiration religieuse dans le cadre architectural pour lequel elles furent primitivement conçues $»^{2}$. Au nom de l'association, Camille Enlart se joignit à l'anathème que la ruine de Reims avait suscité à travers le monde. Il exprima ainsi à Jean-Baptiste Langlet, le maire de la ville qui avait accueilli la Société en mai 1913, toute sa «sympathie, profondément indigné de l'acte de cruauté stupide qui cause [...] un deuil éternel dans le monde civilisé ». Il fit part également au cardinal Louis-Joseph Luçon, archevêque de Reims, de son émotion "particulièrement cruelle à la nouvelle de l'attentat infâme »3 . Ce ressentiment 
s'accompagna, à partir de février 1915, d'une nouvelle prise de position: l'excommunication, véritable bannissement parmi les rangs de l'association, des membres «allemands et austro-hongrois, non comme ennemis et pour la durée de la guerre, mais à toujours, comme solidaires des crimes de droit commun $»^{4}$. C'est avec un engagement tout aussi radical, dénué de compromis, que Camille Enlart organisa au printemps 1915, dans les salles du musée de Sculpture comparée, la première exposition de propagande à visée anti-germanique, véritable prototype du genre.

3 À cette date, le musée de Sculpture comparée, créé en 1879 par Eugène Viollet-le-Duc, relevait directement de la commission des Monuments historiques. Au sein de l'ancien palais du Trocadéro, cette institution rassemblait plusieurs milliers de moulages en plâtre, à grandeur, de la quintessence de la sculpture monumentale française et étrangère, du XII ${ }^{e}$ au XIX ${ }^{e}$ siècle.

Depuis sa nomination en 1903 au poste de directeur du musée, Camille Enlart avait entrepris de réorganiser les collections de manière à mettre en valeur, de façon explicite, les moulages de sculpture française. Il leur accordait l'essentiel des majestueuses galeries du palais et reléguait les exemples tirés de l'Antiquité et la sculpture étrangère, à l'exception de la sculpture flamande, aux espaces secondaires que constituaient les galeries extérieures du palais côté Seine, vitrées depuis l'Exposition universelle de 1900 (fig. $\mathbf{n}^{\circ} \mathbf{1}$ ). Le principe de comparaison entre la sculpture française et étrangère qui, jusqu'alors, avait été un des axes majeurs de l'arrangement des collections était donc profondément modifié, supplanté par une incontestable vision hexagonale. Cette inclination était d'autant plus sensible que Camille Enlart souhaita réserver les nouvelles acquisitions du musée aux seuls moulages de sculpture française ou «à des séries de sculptures exécutées à l'étranger par des artistes français, depuis les monuments des Croisés en Palestine, en Syrie et en Chypre jusqu'aux bustes de nos sculpteurs du XVIII ${ }^{e}$ siècle $»^{5}$. 
Figure 1

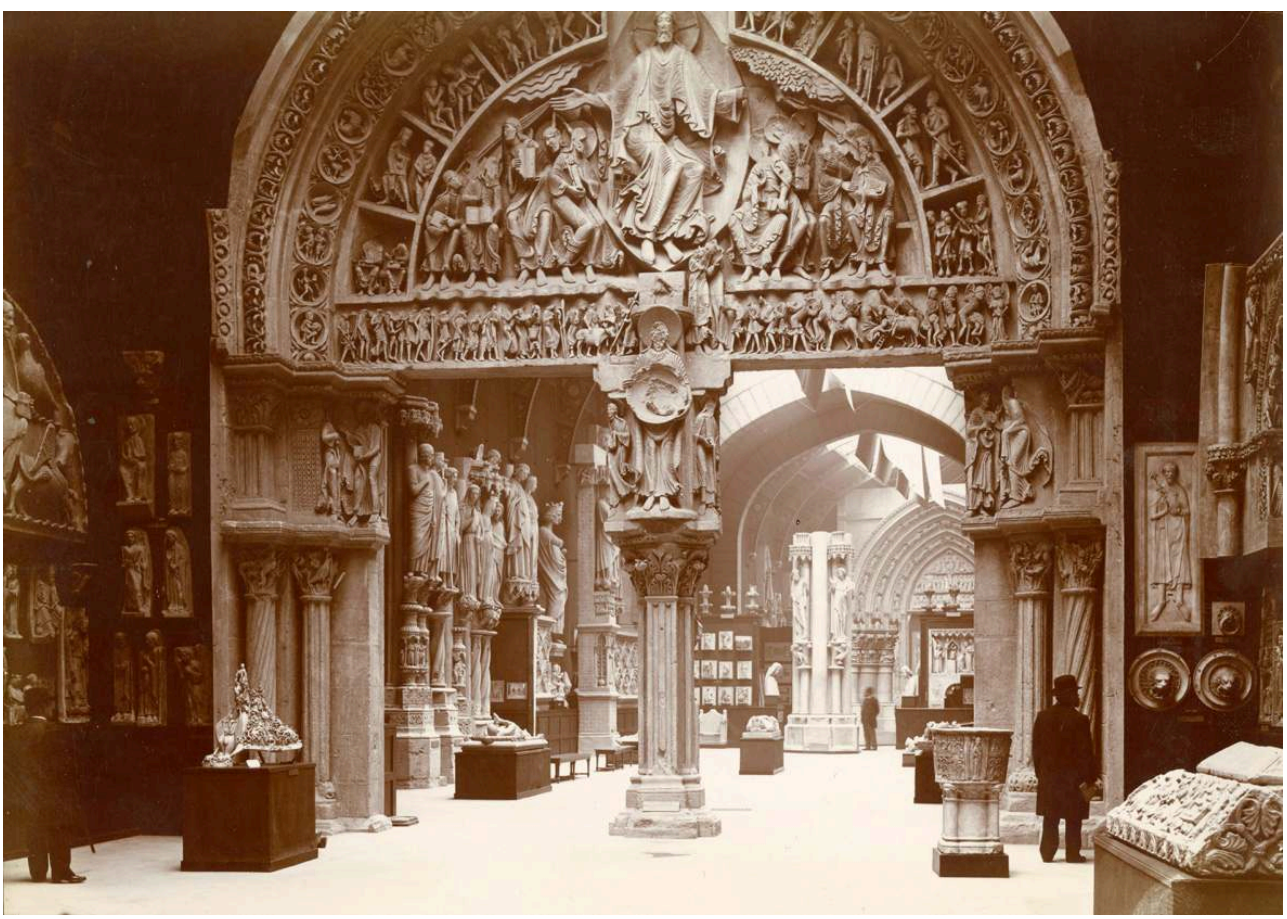

Musée de Sculpture comparée - Aile Paris - Salle A, début du XXe siècle. Au premier plan à gauche : le moulage du pied de candélabre de l'église Saint-Remi, Reims.

(c) Archives du musée des Monuments français/CAPA.

5 Le redéploiement des collections reflétait aussi les courants de pensée de l'École française qui, dans le sillon creusé par des historiens de l'art tels qu'Émile Mâle ou André Michel, portait en elle les dogmes de l'idéal français et de nation-modèle. Camille Enlart adhérait d'autant plus à ces idées qu'il en était lui-même l'un des principaux promoteurs à travers ses recherches et écrits sur le rayonnement de l'art gothique ou le patrimoine monumental de la France septentrionale ${ }^{7}$. La Guerre devait exalter ses sentiments nationalistes et identitaires comme le montre la création, en 1916, de la Société pour la défense de l'art français, fondée et présidée par Camille Enlart afin de «défendre nos traditions d'art français contre l'influence étrangère et d'organiser la renaissance de l'art régional $»^{8}$.

6 Ainsi, au moment où éclatait le conflit, le musée de Sculpture comparée, reconsidéré dans ses contours et son sens profond, doté à sa tête d'un homme de conviction à la fougue patriotique, était-il déjà apte à servir la cause des monuments historiques et la propagande française. Une correspondance en date du 4 août 1914, adressée par Camille Enlart à Albert Dalimier, sous-secrétaire d'État aux Beaux-Arts (14 juin 1914 16 novembre 1917), est exemplaire du caractère impétueux de son engagement. Il confie dans ce document qu'il est de «son devoir (...) de chercher à utiliser pour la Défense Nationale l'établissement confié à [sa] direction » et propose que les galeries du Trocadéro affectées au musée de Sculpture comparée soient transformées en ambulance. «Elles s'y prêtent parfaitement », écrit-il, « étant très aérées, très éclairées, munies de stores contre le soleil et d'une bonne chaufferie». Sans même attendre l'agrément de sa hiérarchie, soutenu par Jules Roussel, conservateur adjoint du musée, il fait ainsi libérer les galeries principales des moulages susceptibles d'entraver son projet par les trois hommes de son personnel de surveillance non mobilisés : le gardien 
$1^{\mathrm{e}}$ classe Durupt, infirmier retraité, le gardien-chef Crucifix et le brigadier Rigaut (fig. $\mathbf{n}^{\circ}$ 2). Quant aux espaces secondaires, peuplés de moulages de sculptures étrangères, ils accueillirent en octobre 1914 les ouvroirs de dames bienfaitrices de la haute société, la comtesse de Ricci et la duchesse de Talleyrand. Dans ces ouvroirs, véritables ateliers improvisés au pied du moulage de la monumentale statue équestre du Colleone, des ouvrières s'affairaient notamment à la confection de vêtements destinés aux troupes? .

Figure 2

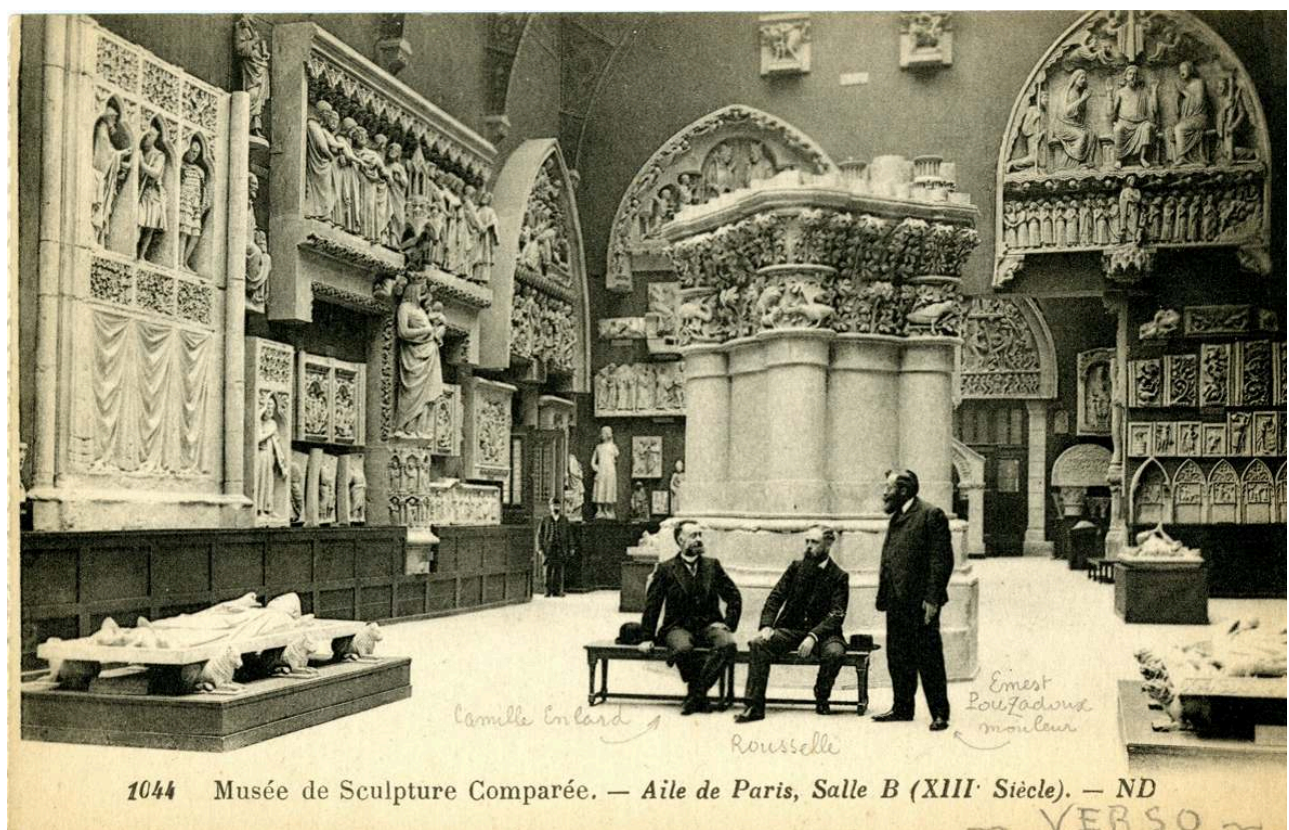

Musée de Sculpture comparée - Aile Paris, début du XXe siècle. De gauche à droite : Camille Enlart, Jules Roussel et Charles Édouard Pouzadoux.

(c) Archives du musée des Monuments français/CAPA.

7 Aucune suite ne fut donnée à la proposition d'infirmerie de Camille Enlart. Implanté sur la colline de Chaillot, le Trocadéro constituait une cible privilégiée pour l'aviation et l'artillerie ennemies; établir un hôpital sous les verrières des galeries, portées à 13 mètres de haut, fut considéré trop dangereux ${ }^{10}$. S'ajoutaient aussi les coûts exorbitants et la difficulté qu'aurait représenté le chauffage des galeries. À cet effet, tout au long du conflit, le musée fut chaque année fermé cinq mois durant, par mesure d'économie, entre le $1^{\mathrm{er}}$ novembre et le $1^{\mathrm{er}}$ avril. Le reste de l'année, l'ouverture de l'intégralité de ses salles fut empêchée en raison d'un nombre insuffisant de gardiens ${ }^{11}$. C'est ainsi qu'en mars 1915 fut officialisé le principe d'une ouverture partielle : tous les jours sauf le lundi et le jeudi pour les salles et la bibliothèque de l'aile Paris, tous les jeudis pour les salles de l'aile Passy - le lundi étant le jour de fermeture hebdomadaire du musée. Ces dispositions furent suivies semble-t-il de manière chaotique, la préférence ayant toujours été accordée à l'ouverture des salles de l'aile Paris (fig. $\mathbf{n}^{\circ} \mathbf{3}$ ). 


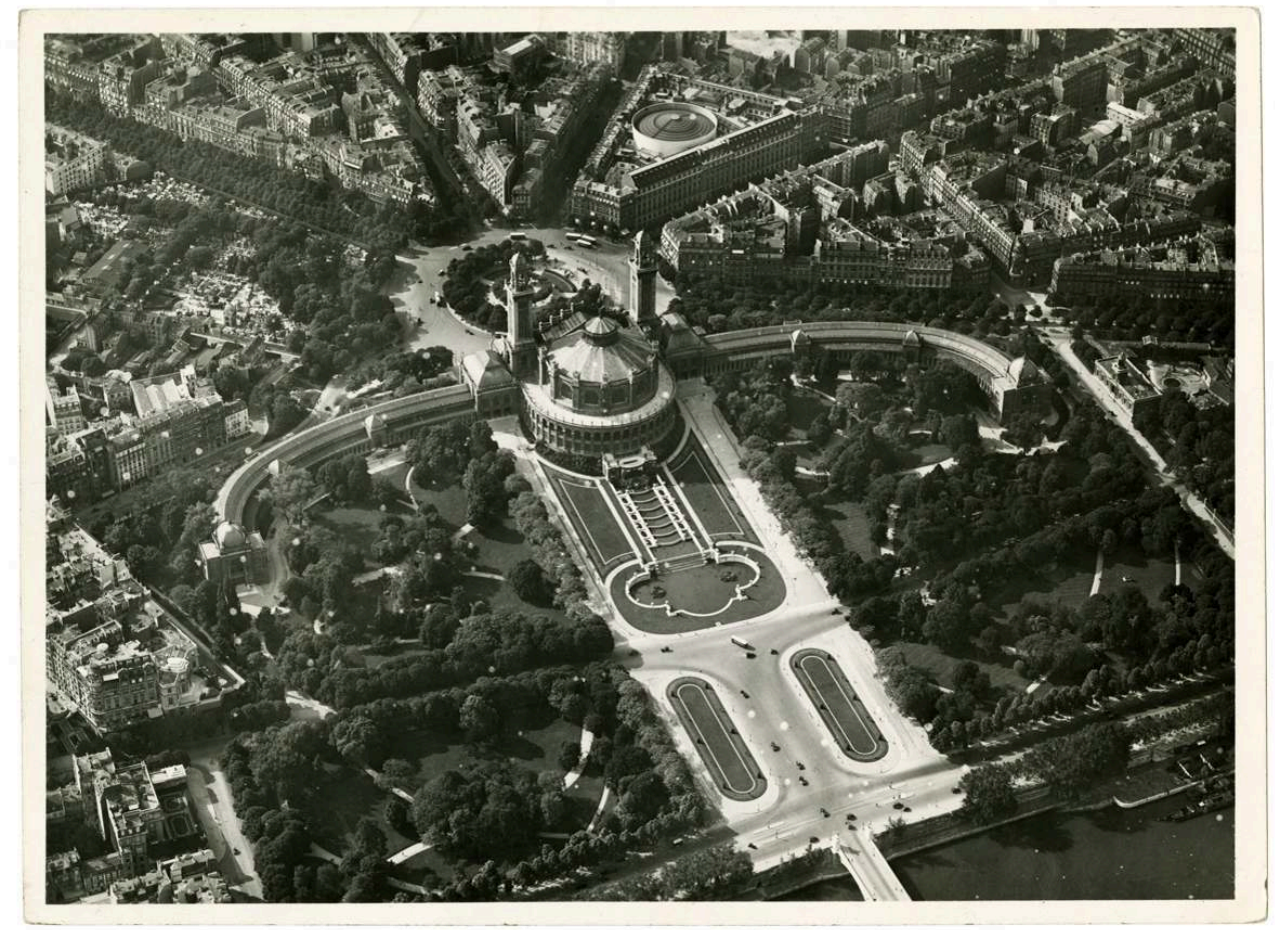

Vue aérienne du palais du Trocadéro, n.d. [avant 1935]. À gauche l'aile Passy ; à droite l'aile Paris. (c) Cie aérienne française. (c) Fonds Carlu. SIAF/Cité de l'architecture et du patrimoine/Archives d'architecture du XXe siècle.

Conforté dans sa mission première, le musée de Sculpture comparée rassembla ses forces en mai 1915, avec la création par Camille Enlart de la première exposition de photographies de monuments dévastés par la guerre. Organisée dans une des travées de l'aile Paris du Trocadéro, cette présentation serait restée confidentielle si la presse quotidienne ne s'en était pas faite timidement l'écho. Les documents d'archives conservés au musée des Monuments français se rapportant à l'opération sont en effet remarquablement peu diserts : à notre connaissance, il n'existe aucune liste précise des photographies exposées et les pièces comptables qui couvrent la période 1914-1918 ne distinguent aucune acquisition à des fins d'exposition. Elles rendent compte, en revanche, de l'enrichissement des fonds photographiques de la bibliothèque du musée, de plusieurs centaines de clichés aux formats $13 \times 18,18 \times 24$ et $30 \times 40$, acquis auprès des photographes Léon Doucet, Henri Heuzé, de la maison Neurdein Frères et, dans une moindre mesure, de la Section photographique de l'armée (SPA). Ces clichés sont aujourd'hui conservés à la Médiathèque de l'architecture et du patrimoine.

En mai 1915, la Section photographique de l'Armée venait tout juste d'être officiellement créée afin de servir la propagande française et contrer la propagande allemande, passée maitre dans la manipulation des images ${ }^{12}$. Il est indéniable que l'action poursuivie par Camille Enlart s'est inscrite dans cette même stratégie car elle en constitue le premier médium, avant l'Exposition des cuvres d'art provenant des régions dévastées par l'ennemi présentée au Petit Palais à partir de 1916 et à laquelle Camille Enlart prêta son concours, mais aussi les non moins spectaculaires expositions de photographies de guerre organisées au pavillon de Marsan en octobre 1916 et au Jeu de paume l'année d'après ${ }^{13}$ (fig. $\left.\mathbf{n}^{\circ} \mathbf{4}\right)$. L'exposition du Trocadéro fut, à cet effet, qualifiée 
de "préface» par ses contemporains. Elle fut par conséquent perçue comme une manifestation pionnière, un modèle de ce qui pouvait être accompli avec les photographies de guerre qui, au même moment, étaient rassemblées par le soussecrétariat d'État aux Beaux-Arts et les services de l'armée ${ }^{14}$.

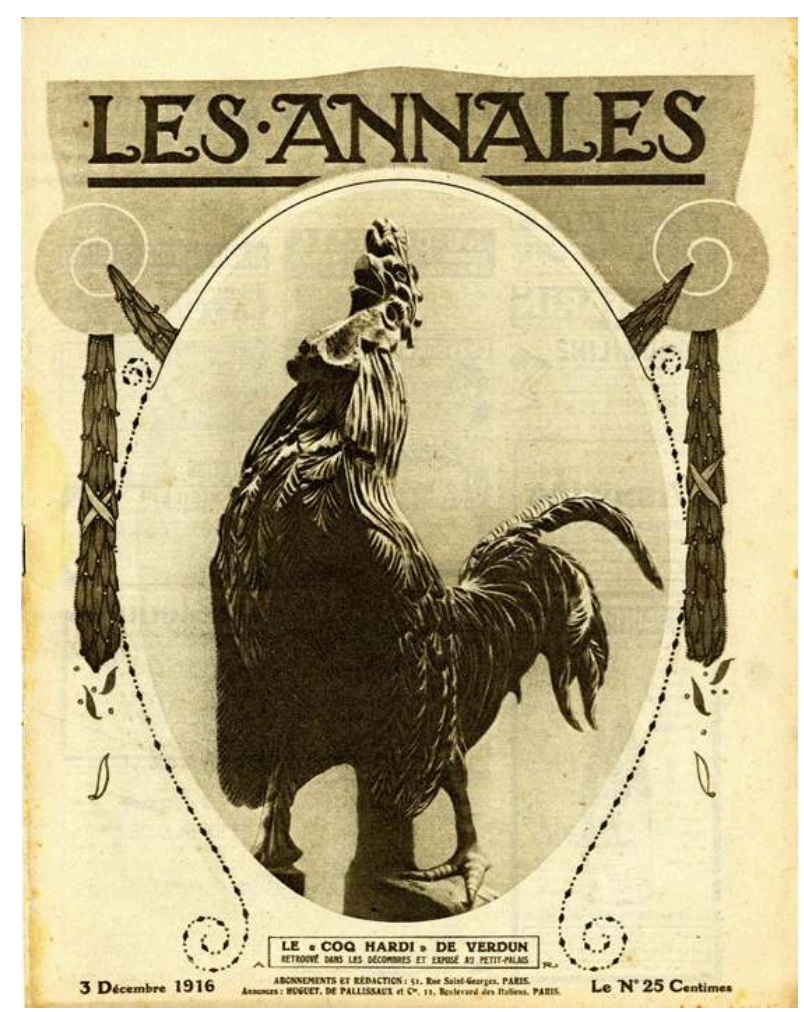

Les Annales du 3 décembre 1916 : l'enseigne du Coq hardi, Verdun, emblème de l'Exposition des Euvres d'art provenant des régions dévastées par l'ennemi (Petit Palais, 1916).

(c) Jean-Marc Hofman.

10 S'agissant principalement de vues de la cathédrale Notre-Dame de Reims après bombardement, de l'hôtel de ville d'Arras, de nombreuses églises rurales de la Marne, de l'Aisne et de la Meuse, il est certain que des photographies acquises pour la bibliothèque furent employées pour l'exposition.

Dans son édition du 28 mai 1915, le Journal des débats politiques et littéraires signale ainsi la présentation au musée de photographies des ruines des cathédrales de Reims et de Soissons, de l'hôtel de ville d'Arras, de Dunkerque. D'autres encore montraient des églises de Champagne, de Lorraine et d'Artois tombées sous le feu de l'artillerie allemande, à l'instar de Maurupt-le-Montois (Marne), de Nettancourt (Meuse) ou de Revigny-sur-Ornain (Meuse). Le quotidien signale encore, à propos de Reims, plusieurs clichés photographiques en passe d'être agrandis pour être exposés, pris par Max Sainsaulieu, architecte des Monuments historiques en charge de la cathédrale ${ }^{15}$. Ces vues révélaient:

...les désastres causés par les bombes dans toutes les parties de l'édifice, depuis les figures des porches au niveau du sol jusqu'au sommet des tours, les arcs-boutants abattus, les pinacles décapités, les roses et les verrières crevées, le revers de la façade, - ce mur unique dans l'art du moyen-âge, cette paroi incrustée de niches et 
de statues, - carbonisés par les bottes de paille dont les Allemands avaient rempli

l'église comme pour préparer l'incendie.

dans le seul cadre de la presse écrite, des actualités cinématographiques ou des conférences animées par des personnalités du monde des arts comme André Michel et Camille Enlart lui-même, mais de manière pérenne, dans le contexte idéal du musée de Sculpture comparée. Avec ses collections de moulages à grandeur de sculpture monumentale, dont certains désormais constituaient l'ultime souvenir des originaux vandalisés ou détruits, le musée de la commission des Monuments historiques conservait l'image d'une France indemne - une nation figée dans sa gloire.

avec lexposition de photographies de monuments détruits, Camille Enlart souhaita ainsi mettre en valeur les moulages des collections qui, du fait de la destruction de leurs modèles, avaient accédé au statut honorable d'œuvre originale. Ces épreuves étaient d'autant plus inestimables que la plupart étaient uniques, le procédé de l'estampage à la terre impliquant la perte du creux lors de l'opération de démoulage. Le statut d'œuvre originale qui anoblissait une partie des collections constitua aussi la principale entrave à la création de nouveaux moules destinés à la réplication d'épreuves. En 1916, la fabrication de creux par l'atelier de moulage du musée de Sculpture comparée, à partir des épreuves exécutées en 1881 de l'Ange dit de saint Nicaise, plus connu sous le nom d'Ange au sourire, et de la reine de Saba fut ainsi refusée en raison du danger de l'opération et de la mobilisation sur le front des hommes compétents pour la mener à bien ${ }^{16}$. Albert Besnard, directeur de l'Académie de France à Rome, en fit lui-même les frais lorsqu'en mai 1918, il souhaita obtenir des moulages de sculpture du Moyen Âge français provenant, en particulier, des cathédrales bombardées, pour les exposer à la villa Médicis. Malgré son insistance auprès du ministre de l'Instruction publique et des Beaux-Arts, les épreuves de Reims ne purent lui être délivrées pour ces mêmes raisons. Ce n'est qu'un an plus tard, le 10 juin 1919, que l'exposition de moulages de sculpture française fut inaugurée à la villa Médicis, en présence de nombreuses personnalités officielles, parmi lesquelles Camille Enlart ${ }^{17}$.

15 Afin de distinguer du premier regard ces épreuves remarquables, Camille Enlart conçut une signalétique spécifique, de couleur verte afin qu'elle se détachât nettement du rouge pompéien des murs du Trocadéro. La documentation se rapportant à ces nouveaux supports est une nouvelle fois très lacunaire, mais elle suffit néanmoins à en mesurer l'effet. Des panneaux de grand format ( 50 x 11 et 40 x 11) portant les messages «Sculpture détruite PAR LES ALLEMANDS " ou "Détruit par les Allemands » furent placardés à proximité des moulages concernés, au nombre desquels l'Ange au sourire de Reims et les semelles de poutres de l'ancienne salle des échevins de l'hôtel de ville d'Ypres (fig. $\mathbf{n}^{\circ}$ ) ). 
Figure 5

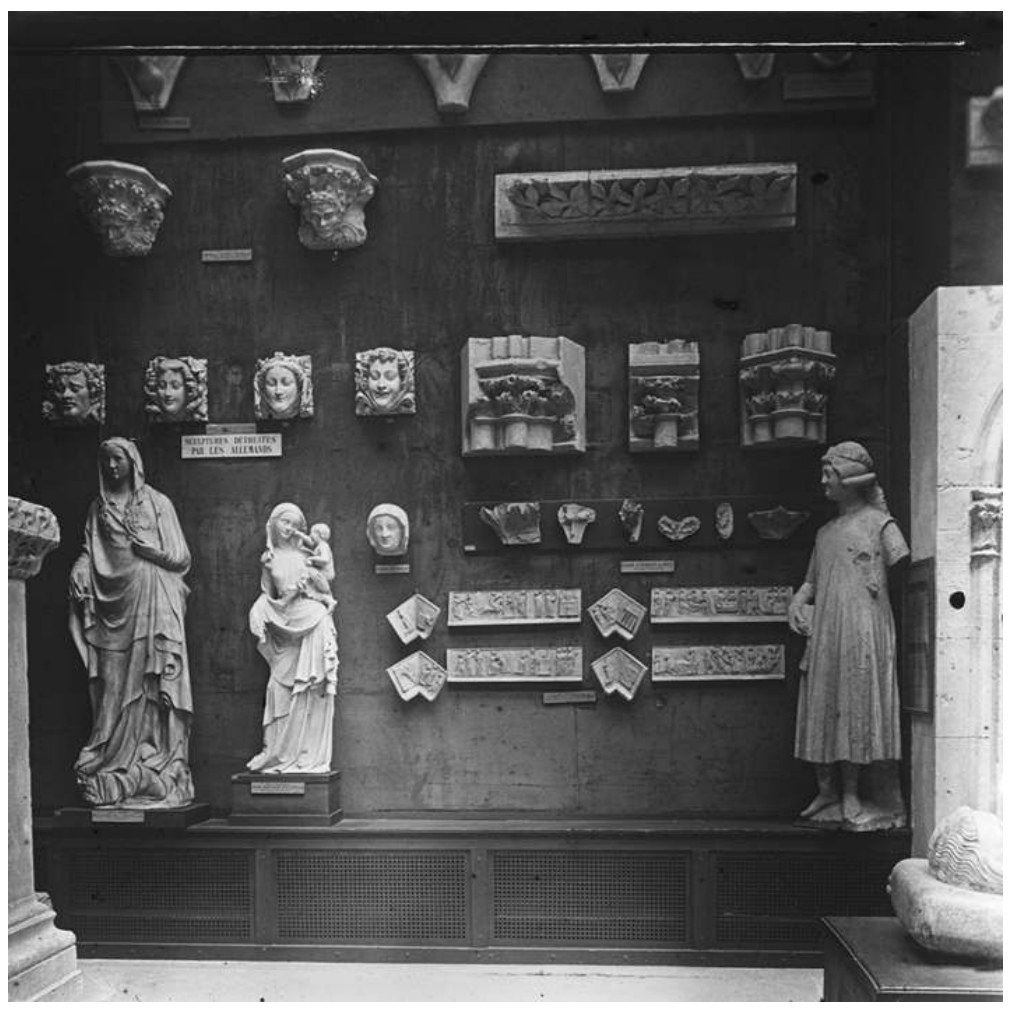

Henri Olivier : Musée de Sculpture comparée - Aile Passy - Salle K. À côté des semelles de poutres de la salle échevinale de l'hôtel de ville d'Ypres figure le panneau « Sculptures détruites par les allemands ». s.d. [vers 1915/1920].

(c) Médiathèque de l'architecture et du patrimoine, Charenton-le-Pont/RMN.

16 Camille Enlart devait cependant ne pas se cantonner au seul premier conflit mondial. Il entendait amener la guerre sur son propre terrain et mener le combat en fustigeant à travers le temps, méthodiquement, l'ennemi tudesque, sempiternel destructeur des $\operatorname{arts}^{18}$. D'autres panneaux de mêmes dimensions furent ainsi réalisés sur lesquels était inscrit « Monuments de la Gaule romaine dévastés par les Barbares du $\mathrm{III}^{\mathrm{e}}$ au Ve siècle » et «Bustes détruits par les Allemands $1870 »\left(\right.$ fig. $\left.\mathbf{n}^{\circ} \mathbf{6}\right)$. Un grand placard ( $80 \times 20$ ) intitulé «Cathédrale et cité de Thérouanne détruites par les Impériaux 1553» fut encore installé à proximité immédiate des estampages en papier du pavement de la cathédrale de Thérouanne (fig. $\mathbf{n}^{\circ}$ ). Cette édifiante chronologie délivrait désormais dans les salles du musée le message que Camille Enlart réservait habituellement à l'auditoire de ses conférences : la "sauvagerie teutonne, en tant qu'elle s'exerce contre les édifices religieux, ne fait guère que tenir en 1914 et 1915 ce qu'elle promettait dès 1870. [...] Elle s'est développée comme se développe un enfant pour devenir un homme $»^{19}$. 
Figure 6

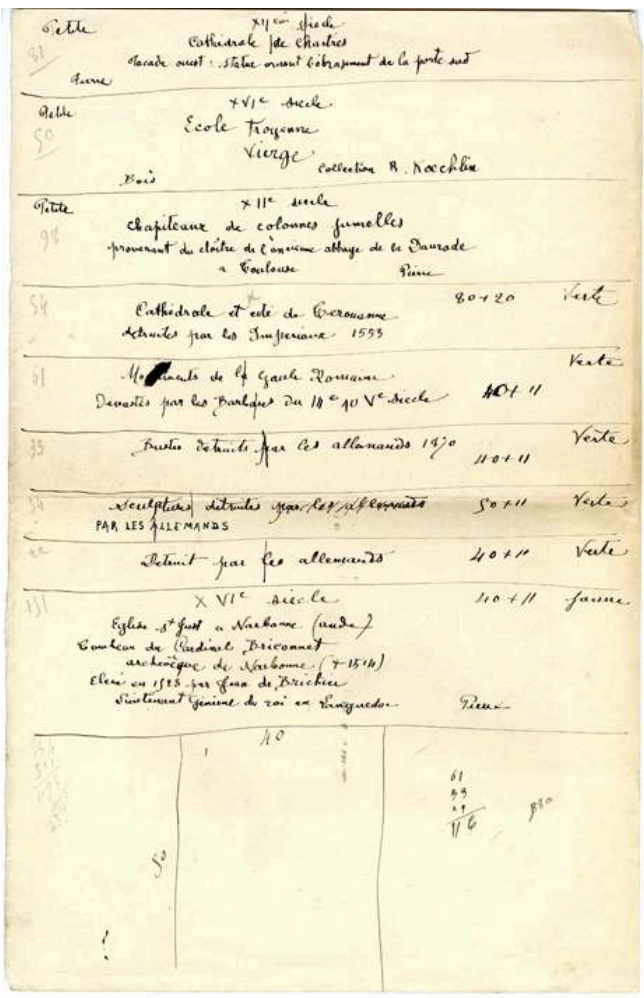

Projet d'étiquettes. Archives du musée des Monuments français/CAPA/MSC 20/Comptabilité 1917.

(c) Archives du musée des Monuments français/CAPA. 
Figure 7

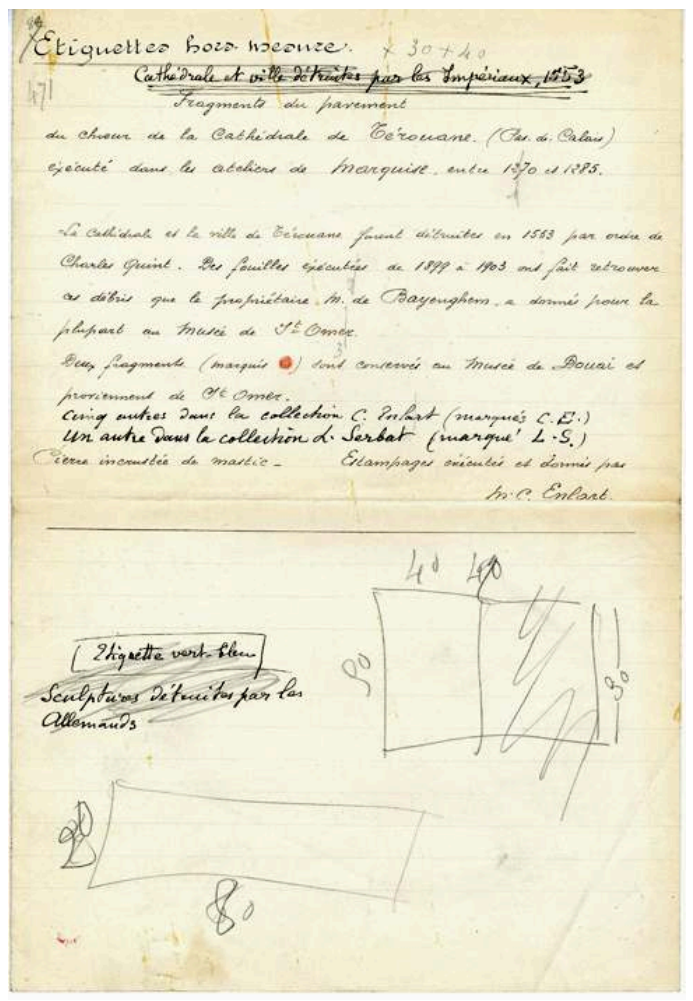

Projet d'étiquettes. Archives du musée des Monuments français/CAPA/MSC 20/Comptabilité 1917.

(c) Archives du musée des Monuments français/CAPA.

17 Cette instrumentalisation du patrimoine national qui élevait le musée du Trocadéro au rang de site mémoriel se distingue en 1916 sous la plume du critique d'art François Thiébault-Sisson :

Et comme il ne faut qu'aucun de ces méfaits ne s'oublie, M. Enlart a fait apposer dans son musée, pour l'édification des visiteurs, des étiquettes très apparentes, peintes en vert clair, qui désigneront désormais les moulages des œuvres d'art détruites par les Allemands non seulement pendant la présente guerre, mais au cours de toutes les guerres antérieures, depuis 1870 jusqu'au quatrième ou cinquième siècle de notre ère, en passant par le seizième que marqua l'invasion par Charles Quint. Sans cette contrepartie, qui nous fait voir notre ennemi national, d'un bout de l'histoire à l'autre, toujours pareil à lui-même, la leçon que le musée fournit risquerait d'être incomplète. En opposant au peuple de France, éternel créateur, l'éternel destructeur qu'est le Teuton, elle magnifiera le premier, elle vouera à l'exécration universelle le second - et ce sera justice ${ }^{20}$.

L'imprécation de Camille Enlart était irrévocable. La guerre achevée, il tint en effet à maintenir dans les salles cette signalétique si singulière. Ainsi qu'il l'exprime en 1919, dans son sens profond, le musée de Sculpture comparée se devait plus que jamais d'assumer un devoir moral devant l'histoire nationale et être "d'un enseignement complet. Il témoigne du génie de nos pères ; il dira aussi le crime des éternels ennemis de la civilisation ${ }^{21}$.

Avec ces nouvelles dispositions, Camille Enlart contribuait, à sa manière et avec les moyens qui étaient les siens, à l'œuvre collective d'union nationale que réclamait l'intrusion de la guerre dans la société civile. Il était parvenu à instaurer dans les salles du musée de Sculpture comparée un climat fédérateur empreint de respect, un silence 
propre à signifier l'indignation des destructions, le seul capable aussi de laisser percer le bruit des combats qui se jouaient au nord et à l'est de la capitale. C'est le message que délivre une chronique intimiste intitulée Lettre à une amie, publiée dans le quotidien Le Gaulois :

Dans cette salle du musée enveloppée d'une atmosphère de recueillement, d'étude, où l'esprit reste naturellement froid, où l'on est porté à juger sans haine, éclate le caractère effroyable de cette guerre [...] Vous verrez là, vous à qui il est interdit d'approcher des « lignes », ce qu'ils ont fait d'Arras, de Soissons ${ }^{22}$.

Le compte rendu d'une visite de la société des Amis des Monuments présidée par Charles Normand, le jour de la Toussaint 1915, confirme ce sentiment que, désormais, le musée du Trocadéro servait de théâtre à «un autre culte des morts - celui des monuments nationaux qui ont souffert ou péri pour la patrie $»^{23}$.

\section{NOTES}

1. - Jean-Marc Hofman est adjoint au conservateur de la galerie des moulages du musée des Monuments français, à la Cité de l'architecture et du patrimoine (Paris). Ses thèmes de recherche portent notamment sur l'histoire du musée de Sculpture comparée et du musée des Monuments français, ainsi que sur le commerce des moulages à travers le monde.

2. - Société des amis des cathédrales, Bulletin annuel, 1913, 3.

3. - La protestation des Amis des cathédrales est reproduite dans l'édition du 2 octobre 1914 du Journal des débats politiques et littéraires. Au sujet des réactions internationales: LANDRIEUX, Maurice. La Cathédrale de Reims : un crime allemand. Paris : Librairie Renouard, H. Laurens, éditeur, 1919, p. 208-229 ; BOUHELIER (de), Georges. Les Allemands destructeurs de cathédrales et de trésors du passé (documents officiels), mémoire relatif aux bombardements de Reims, Arras, Senlis, Louvain, Soissons, etc. Paris : Hachette et Cie, 1915.

4. - Société des amis des cathédrales, Bulletin des années 1915-1919, 17-18. Même après guerre, en 1919, une modification des statuts de la société portant sur l'obligation de produire un certificat de nationalité pour en devenir membre fut approuvée à l'unanimité.

5. - ENLART, Camille. Le musée de Sculpture comparée du Trocadéro. Collection Les grandes Institutions de la France. Paris : H. Laurens, Éditeur, 1911, p. 8-9.

6. - MICHEL, André. Histoire de l'art depuis les premiers temps chrétiens jusqu'à nos jours. Tome II, 1ère partie: formation, expansion et évolution de l'art gothique. Paris: Armand Colin, 1906, VIII : "La France en toutes choses donna le ton. Qu'il s'agisse d'architecture, de sculpture ou de miniature, tous les peuples qui avaient déjà, chacun pour son compte, élaboré un style national, rencontrèrent sur leur route l'idéal français, et voulurent le suivre. "

7. - PAUL, Anne-Cécile. "Camille Enlart». Dans Dictionnaire critique des historiens de l'art actifs en France de la Révolution à la Première Guerre mondiale. SÉNÉCHAL, Philippe, BARBILLON, Claire, dir. Paris : site web de l'INHA, 2009. URL : http://www.inha.fr/spip.php?article2309.

8. - Le Temps du $1^{\mathrm{er}}$ août 1916, p. 2. Dans cette même veine, André Michel dispensa à Paris plusieurs conférences sur le thème de l'art français après la guerre. Il y exprimait le souhait que les « artistes fussent des représentants de leur province, de leur pays et de leur race » (Le Gaulois, 24 février 1917). 
9. - À l'exception des légendes datées d'un jeu de clichés photographiques de Charles Lansiaux, conservés à la Bibliothèque historique de la Ville de Paris, nous n'avons trouvé aucune mention des ouvroirs organisés au sein du musée. Un seul cliché, daté du 5 octobre 1914, se rapporte à l'ouvroir de la duchesse de Talleyrand ; trois autres, datés du 7 octobre 1914, portent mention de l'ouvroir de la comtesse de Ricci et se déroulent distinctement dans les salles du musée.

10. - Archives du musée des Monuments français, CAPA/MSC 3/Fermeture Musée pendant la guerre 1915-1916: le projet de mise à disposition du musée de Sculpture comparée pour y installer une ambulance fut porté à la connaissance du ministre de la guerre par lettre du soussecrétaire d'État aux Beaux-Arts en date du 6 août 1914. Il est semble-t-il définitivement écarté en mars 1915, Camille Enlart signalant dans une correspondance du 15 mars, avoir fait procéder à « la remise en place des objets qui avaient été garés en vue de l'utilisation de nos galeries par le Ministère de la guerre ".

11. - Archives du musée des Monuments français, CAPA/MSC 3/Fermeture Musée pendant la guerre 1915-1916 : en 1915, Camille Enlart écrit à son administration que sur les neuf gardiens qui assuraient la sécurité des œuvres, cinq sont portés manquants, par suite de mobilisation et de décès.

12. - GUILLOT, Hélène. " La section photographique de l'armée et la Grande Guerre ». Revue historique des armées [En ligne], 258, 2010, mis en ligne le 26 février 2010 : URL : http:// rha.revues.org/6938.

13. - Camille Enlart comptait parmi les membres du comité de patronage de l'exposition du Petit Palais. Voir: Exposition d'œuvres d'art mutilées ou provenant des régions dévastées par l'ennemi. Paris: Impr. D. Niestlé, 1916, p. 3. L'exposition du Trocadéro anticipe sur deux manifestations de photographies majeures et particulièrement soignées sur le plan muséographique : l'Exposition de photographies de guerre des armées alliées présentées en octobre 1916, au pavillon de Marsan et l'Exposition interalliée de photographies de guerre, présentée en 1917 au Jeu de paume.

14. - Le terme de "préface » est employé dans un article intitulé «Un témoignage de la Kultur germanique » publié dans l'édition du 24 mai 1915 du quotidien Le Petit Parisien. Présente dans les intentions du fondateur du musée de Sculpture comparée, Eugène Viollet-le-Duc, la photographie connut sous le directorat de Camille Enlart un formidable développement. De 40000 photographies en 1907, la collection de la bibliothèque du musée de Sculpture comparée atteint 125000 clichés à la mort de Camille Enlart, en 1927. Sur ce sujet, consulter: LANCESTREMÈRE, Christine et POLACK, Emmanuelle. «La photographie au musée de Sculpture comparée ». Revue de l'art, $\mathrm{n}^{\circ} 181 / 2013-3$, p. 63-69.

15. - Archives du musée des Monuments français, CAPA/MSC 20/Comptabilité 1915. Une facture de la Maison Neurdein frères en date du 24 juin 1915 mentionne l'acquisition de clichés Sainsaulieu : 111 épreuves 13 x 18 et 111 épreuves 30 x 40 de la cathédrale de Reims. Ils furent inventoriés au musée sous les $n^{\circ} 57970$ à 58080 pour les premiers; sous les n 58082 à 58192 pour les seconds.

16. - Sur l'histoire des moulages de Reims et le contexte d'exécution des creux pendant la guerre par l'atelier du musée dirigé par Charles Édouard Pouzadoux : HOFMAN, Jean-Marc et GUILHEM, Élodie. «Die Abgüsse nach Skulpturen der Kathedrale Notre-Dame in Reims in den Sammlungen des Musées des Monuments français ». Der Naumburger Meister. Bildhauer und Architekt im Europa der Kathedralen. Band 1, Naumburg (Allemagne), 2011, p. 316-323. En 1915, un moule à bon creux du pied de candélabre de Saint-Remi de Reims avait été exécuté par l'atelier du musée. Dans un contexte d'exaltation patriotique lié à l'Exposition des œuvres d'art provenant des régions dévastées par l'ennemi, le refus de réaliser de nouveaux moules fit l'objet de violentes chroniques par l'écrivain Joséphin Péladan. Les mouleurs mobilisés sur le front étaient Ernest Velten, Charles Thierry et André Grandvoinet. Blessé sur le Chemin des Dames le 5 mai 1917, André Grandvoinet devait succomber le 22 mai 1917. Voir Archives de Paris, Registre D4R1/1070 nº matricule 3359. 
17. - Gazzetta ufficiale del regno d'Italia, $\mathrm{n}^{\circ} 138,11$ juin 1919, p. 1608. Les archives relatives au directorat d'Albert Besnard conservées à la villa Médicis montrent qu'une première série de moulages de sculpture française fut accordée à titre gracieux en juin 1918. Moins d'un mois plus tard, Albert Besnard souhaita compléter cette sélection par une seconde série comprenant l'Ange au Sourire, la reine de Saba et les Fenêtres des Musiciens de Reims. Comme il n'en existait aucun moule, cette seconde série fut ajournée et reportée à la fin des hostilités. L'épouse d'Albert Besnard s'impliqua particulièrement dans l'organisation de cette exposition qui poursuivait un double objectif : servir la propagande et l'histoire de l'art.

18. - Deux séries de panneaux furent exécutées. La première série, qui se rapporte aux monuments détruits lors du conflit en cours, est installée dans les salles dès le printemps 1915 ; la seconde, avec ses perspectives historiques, est plus tardive. Elle est annoncée dans un document conservé aux archives des musées nationaux que l'on peut dater d'après son contenu de novembre 1915: Archives des Musées nationaux, 5HH10(4) - ENLART, Camille. "Les accroissements du musée de Sculpture comparée ", s.d. Archives du musée des Monuments français, CAPA/MSC 20/Comptabilité Exercice 1916 : Des factures du peintre Loris Labbé datées du 8 mai 1916 concernent l'exécution de messages sur panneaux et étiquettes vertes correspondant aux deux séries. PASSINI, Michaela. La fabrique de l'art national. Le nationalisme et les origines de l'histoire de l'art en France et en Allemagne 1870-1933, Collection Passages, vol. 43. Paris : Éditions de la Maison des sciences de l'Homme, 2012, p. 202. Sur le plan de l'histoire de l'art, dans son étude, Michaela Passini souligne encore la virulence peu commune avec laquelle Camille Enlart combattit le terme de "gothique", dont la guerre avait rendu inacceptables les résonances germaniques.

19. - NARFON (de), Julien. « Les Ennemis des cathédrales ». Le Figaro du 19 mai 1915, p. 5. Au cours de la guerre, Camille Enlart présenta de nombreuses conférences avec projections sur ce thème : Le vandalisme allemand en France le 22 janvier 1916, Le Vandalisme en Artois le 26 janvier 1916, Les destructions allemandes le 10 novembre 1917, etc.

20. - THIEBAULT-SISSON, François. « La réouverture des musées. Les remaniements du musée du Trocadéro ». Le Temps, du 25 février 1916. Au sujet de la transformation du musée en lieu de mémoire: FLOUR, Isabelle. «Style, nation, patrimoine: du musée de Sculpture comparée au musée des Monuments français (1879-1937) ». Stratégies identitaires de conservation et de valorisation du patrimoine. NÉMERY, Jean-Claude, RAUTENBERG, Michel et THURIOT, Fabrice (dir.) Paris : L'Harmattan, 2008, p. 37-38.

21. - ENLART, Camille. «Le musée de Sculpture comparée du Trocadéro pendant la guerre ». Les Arts, $n^{\circ} 179,1919$, p. 18-19. POULOT, Dominique. «Le musée d'histoire en France entre traditions nationales et soucis identitaires ». An. mus. paul., vol. 15, n² 2, São Paulo, juillet-décembre, 2007, p. 296-316. URL: http://dx.doi.org/10.1590/S0101-47142007000200021. Dominique Poulot souligne que l'appel à l'émotion du souvenir est encore attaché au musée documentaire créé par Henri Blanc pendant la guerre. Avec l'exemple du musée de Sculpture comparée, il participe du " processus de l'identité nationale dans le sentiment de la perte d'un héritage commun ».

22. - Le Gaulois du 22 septembre 1915.

23. - Le Petit Parisien du 2 novembre 1915. 


\section{RÉSUMÉS}

Cet article apporte un éclairage sur la situation du musée de Sculpture comparée, à Paris, et l'implication de son directeur, Camille Enlart, au cours de la Première Guerre mondiale. Dans un contexte d'exaltation nationaliste, Camille Enlart organisa en 1915 la première exposition de photographies dédiées aux destructions architecturales et artistiques, parmi les collections de moulages en plâtre de monuments historiques exécutées dans le dernier tiers du XIX ${ }^{e}$ siècle. À l'aide d'une signalétique "offensive », il souligna la valeur archéologique des moulages dont les originaux avaient été partiellement ou entièrement détruits. À défaut de combattre dans les tranchées, Camille Enlart mena la guerre à l'intérieur du musée : il utilisa les armes culturelles et identitaires pour combattre l'ennemi et soutenir la propagande française.

This article deals with the situation of the museum of Comparative sculpture in Paris and the particular implication of its director Camille Enlart during the First World War. In the context of an exaltation of nationalism, he organized in 1915, among the plaster cast collections of French historical monuments executed in the last third of the nineteenth century, the first exhibition of photographs dedicated to architectural and artistic destructions. He pointed out with 'offensive' notices the archeological value of the casts of the collections the originals of which were entirely or partly destroyed. Without being in the trenches, Camille Enlart led a war from inside the museum, using cultural and identity weapons to fight the enemy and to support French propaganda.

\section{INDEX}

Mots-clés : Camille Enlart, musée de Sculpture comparée, Trocadéro, moulage, nationalisme, propagande, SPA, Albert Besnard

\section{AUTEUR}

\section{JEAN-MARC HOFMAN}

Adjoint au conservateur de la galerie des moulages, Cité de l'architecture et du patrimoine / musée des Monuments français jmhofman@citechaillot.fr 